\title{
Renal Colic: Epidemiological, Clinical Etiological and Therapeutic Aspects at the Urology Department of the National Reference General Hospital of N'Djamena (Chad)
}

\author{
Mahamat Ali Mahamat ${ }^{*}$, Alkadri Diarra ${ }^{2}$, Amadou Kassogué ${ }^{3}$, Divine Eyongeta ${ }^{4}$, \\ Vadandi Valentin'1, Ndormadjita Allah-Syengar'1, Koldimadji Mingué1, Ramat Wakamadja1, \\ Moussa Kalli1, Okim Ahmat', Kimassoum Rimtebaye ${ }^{1}$ \\ ${ }^{1}$ University of N'Djamena and the National General Reference Hospital of N'Djamena (NGRH), N'Djamena, Chad \\ ${ }^{2}$ Urology Department, Luxembourg University Teaching Hospital of Bamako, Bamako, Mali \\ ${ }^{3}$ Urology Department, Pr Bocar Sidi Sall University Teaching Hospital of Kati, Kati, Mali \\ ${ }^{4}$ Urology Division, Department of Surgery, Regional Hospital Limbe, Limbe, Cameroon \\ Email: *doctaali@yahoo.fr
}

How to cite this paper: Mahamat, M.A., Diarra, A., Kassogué, A., Eyongeta, D., Valentin, V., Allah-Syengar, N., Mingué, K., Wakamadja, R., Kalli, M., Ahmat, O. and Rimtebaye, K. (2020) Renal Colic: Epidemiological, Clinical Etiological and Therapeutic Aspects at the Urology Department of the National Reference General Hospital of N'Djamena (Chad). Open Journal of Urology, 10, 25-33.

https://doi.org/10.4236/oju.2020.102004

Received: November 19, 2019

Accepted: January 17, 2020

Published: January 20, 2020

Copyright $\odot 2020$ by author(s) and Scientific Research Publishing Inc. This work is licensed under the Creative Commons Attribution International License (CC BY 4.0).

http://creativecommons.org/licenses/by/4.0/

(c) (i) Open Access

\begin{abstract}
Introduction: Renal colic is a medico-surgical emergency in which complicated forms could progress to renal failure and nonfunctional kidney. The objective of our study is to describe the epidemiological, diagnostic and therapeutic aspects of renal colic at the urology department of the NGRH. Methods: This was a prospective study involving 101 patients followed up for renal colic. The study spanned from July 2015 to July 2016 at the urology department of the NGRH. Results: A total of 101 patients suffering from renal colic were enrolled in the study giving a prevalence of $5.1 \%$. The mean age was 38.89 years with a standard deviation of 14.5 years and a sex ratio of 1:3. Flank or lumbar pain of crushing type (45.5\%), constant and of severe intensity $(61.4 \%)$ were the most frequent clinical characteristics. The plain abdominal radiography (Kidney-Ureter-Bladder) coupled with ultrasonography had a $100 \%$ sensitivity for the etiological diagnosis of renal colic. Urinary lithiasis was the most frequent cause with $73.3 \%$. Uroculture revealed a urinary tract infection in 44 patients (43.6\%). The most frequent germ isolated on the culture was Escherichia coli (27.7\%). Renal function was abnormal in 34 patients (33.7\%). Medical treatment was composed of analgesics, NSAIDs and antispasmodic representing 93.1\%. Percutaneous nephrostomy was performed in $6.9 \%$. Pyelolithotomy was the most frequently performed surgical procedure and was done in 11 patients that are $39.3 \%$. Fifty seven patients (56.4\%) got healed without sequelae; 6 cases (5.9\%) of recurrence and 2
\end{abstract}


deaths (2\%) were recorded. Conclusion: Renal colic is common in urology. Urinary lithiasis was the major cause in our series. Renal function could be endangered in case of late management.

\section{Keywords}

Renal Colic, Lithiasis, Bilharziasis, NGRH, Chad

\section{Introduction}

Renal colic is an acute flank or lumbar pain resulting from the sudden stretching of an obstructed pyelocalyceal system irrespective of the cause [1]. It usually results from urinary lithiasis. It is also frequent in patients received at the urology department. The prevalence of renal colic has been reported to be variable in Africa: in Senegal TOURE et al., reported a frequency of 0.39\% [2], while in Burkina Faso, Kambou et al., reported 6.6\% [3]. It has been reported that pains crises occur mostly during the dry season, and at night or very early in the morning [4]. The initial treatment must be done early and as an emergency before investigating the etiology [5]. It is a medico-surgical emergency. Renal colic could have major repercussions which could evolve towards renal failure, nonfunctional kidney or septicemia. The objectives of this study were to describe the epidemiological, clinical, etiological and therapeutic aspects of renal colic at the urology department of the NGRH.

\section{Patients and Methods}

It was a prospective and descriptive study carried out over a period of twelve months running from the $31^{\text {st }}$ of July 2015 to the $30^{\text {th }}$ of July 2016 at the Emergency and Urology Departments of the NGRH. A total of 101 cases were enrolled during the period of study. Included in our study, were patients who presented renal colic with an established diagnosis and treatment. Excluded in our study, were patients who presented lumbar pain not typical of renal colic. Study variables were age, sex, risk factor, antecedents, clinical characteristics of the pain, feeding habits, paraclinical (ultrasound-plain abdominal x-ray KUB, intravenous urography, computed tomography urography, urine culture with sensitivity tests and urinary sediment), emergency then definitive treatment, and postoperative follow-up. Data were collected and analysed using the SPSS 11.0 software. Calculations were done using proportions and mean, and statistical significance was considered with $\alpha=5 \%$.

\section{Results}

\subsection{Epidemiological Data}

\subsubsection{Incidence}

During the study period, we received 1946 patients at the urology department of 
the NGRH among which 101 presented and were managed for renal colic giving an incidence of $5.1 \%$. The distribution of age is shown in Figure 1. The mean age of our patients was $38.89 \pm 14.5$ years (range 17 to 72 years). The age range 21 - 40 years was mostly involved with 45 patients giving $44.6 \%$.

Sex:

Majority of the patients were males; 57 men (56.4\%) and 44 women (43.6\%) with a sex ratio of 1:3.

\subsubsection{Climatic Zone}

More than half of our patients (59 patients that is 58.4\%) lived in the sahelian zone (Figure 2), renal colic was observed throughout the year with a peak occurring in the hottest months: March, April and May representing 8.9\%, 20.8\%, and $35.6 \%$ of cases respectively. In our series, 64 patients (63.4\%) lived in urban areas while 37 patients (36.6\%) lived in rural areas.

Feeding habits of our patients composed essentially of milk and other dairy products (64 patients giving 63.4\%), followed by cereals and red meat.

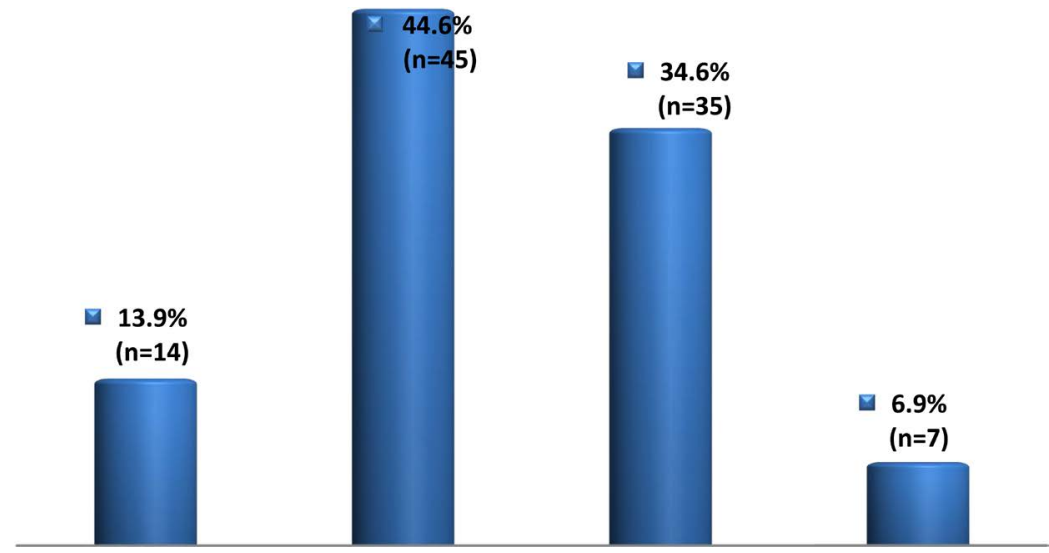

Figure 1. Distribution of patients according to age.

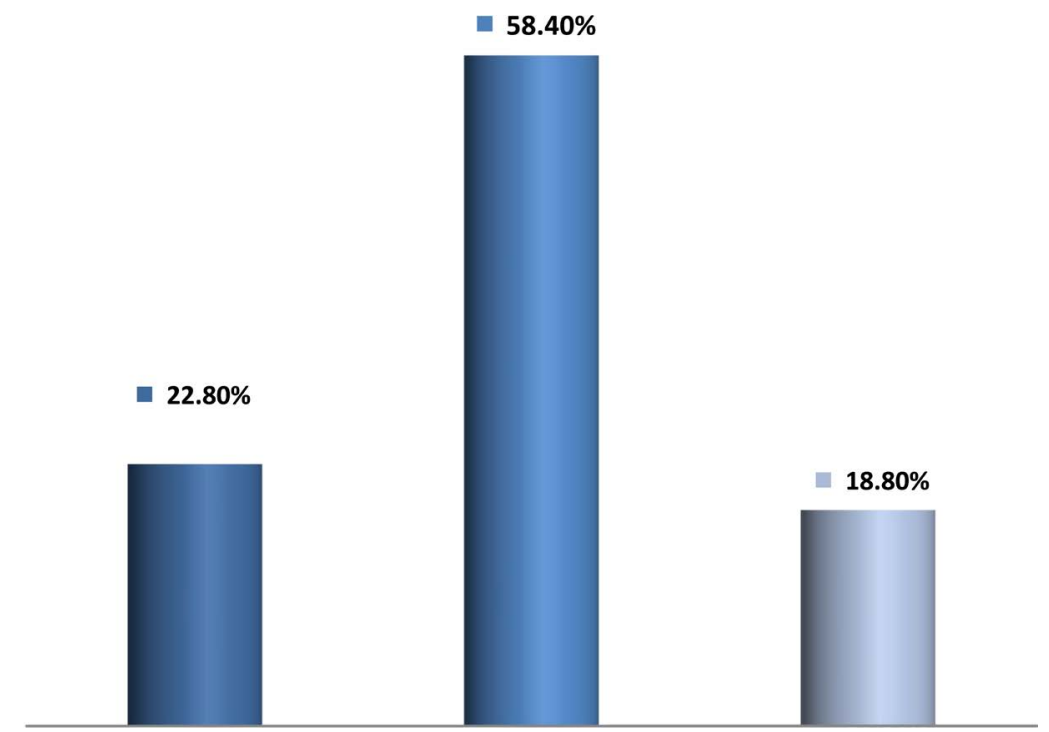

Figure 2. Distribution of patients according to the climatic zones of Chad. 


\subsection{Mode of Admission}

Half of our patients came from the emergency department (51 patients that are $50.5 \%$ ) (Figure 3). Majority of our patients presented unilateral lumbar pain (92.1\%). Bilateral pain was less frequent in our study. Lumbar pain of crushing type (45.5\%) and severe intensity, constant pain (61.4\%) were the mostly encountered clinical characters. In our series, 74 patients (that are $73.8 \%$ ) had experienced at least one episode of renal colic in the past. Chronic urinary tract infection was the urologic antecedent mostly reported (19 patients that are 18.8\%).

\subsection{Clinical Forms of Renal Colic}

Simple renal colic occurred in 64 patients that are $63.4 \%$, while 19 patients (18.8\%) experienced extremely painful renal colic as depicted in Table 1. All patients did a urine culture and sediment. E. coli was mostly identified (28 patients that are $27.7 \%$ ) followed by eggs of Schistosoma haematobium (7 patients that are $6.9 \%$ ). Plain abdominal (KUB) X-ray was performed in $83.2 \%$ of patients, and revealed a radiopaque calculus in 45 of them giving $44.6 \%$. Hydronephrosis from renal lithiasis was the abnormality mostly found on ultrasound and intravenous urography representing $38.6 \%$ and $51.6 \%$ (16 patients) respectively.

\subsection{Etiology}

Urolithiasis of the upper urinary tract was the major cause of renal colic occurring in 74 patients (73.3\%) followed by sequelae of bilharziasis with resultant
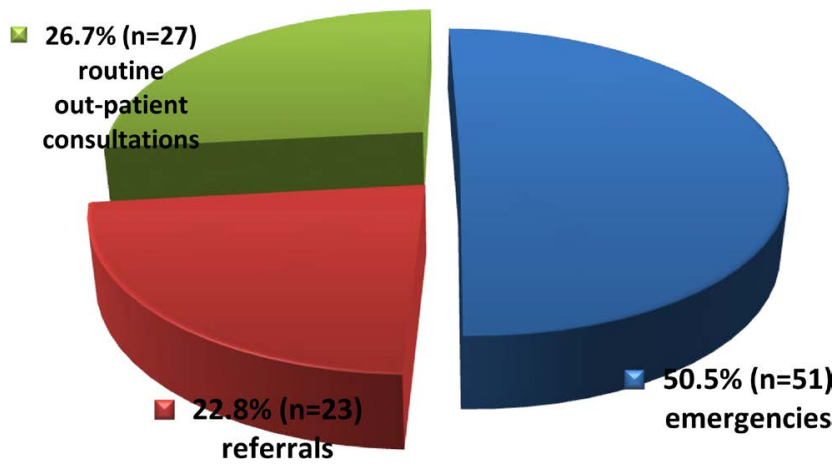

Figure 3. Distribution of patients according to the mode of admission.

Table 1. Distribution of patients according to forms of renal colic.

\begin{tabular}{ccc}
\hline Clinical forms & N & $\%$ \\
\hline Simple renal colic & 64 & 63.4 \\
Extremely painful renal colic & 19 & 18.8 \\
Febrile renal colic & 8 & 7.9 \\
Oligo-anuric renal colic & 7 & 6.9 \\
Renal colic in pregnancy & 3 & 3.0 \\
Total & 101 & 100 \\
\hline
\end{tabular}


ureteral stricture in 7\%.

\subsection{Treatment}

\subsubsection{Medical Treatment}

During pain crisis, 73 patients $(72.3 \%)$ were treated with NSAIDs + analgesics. Ultrasound-guided percutaneous nephrostomy (Figure 4) was performed as an emergency on 7 patients $(6.9 \%)$.

\subsubsection{Surgical Approach}

In our series, 28 patients underwent surgery, and pyelolithotomy was the surgical technique mostly performed (11 patients giving 39.3\%). Nephrectomy was done in 5 patients (17.9\%) as depicted in Table 2.

\subsection{Evolution and Complication}

Out of the 28 operated patients, post-operative course was uneventful in $23(82.1 \%)$. Complications were noted in 10 patients (35.71\%): renal failure in 5 patients (17.86\%), hyperalgic pain in 3 patients (13.04\%) and fever in 2 patients (7.14).

Of the 28 patients operated on, we noted 2 parietal suppurations, 2 cases of fever in post-operative care and 1 case of hyperalgesic pain. 23 patients $(82.1 \%)$ had simple post-operative outcomes.

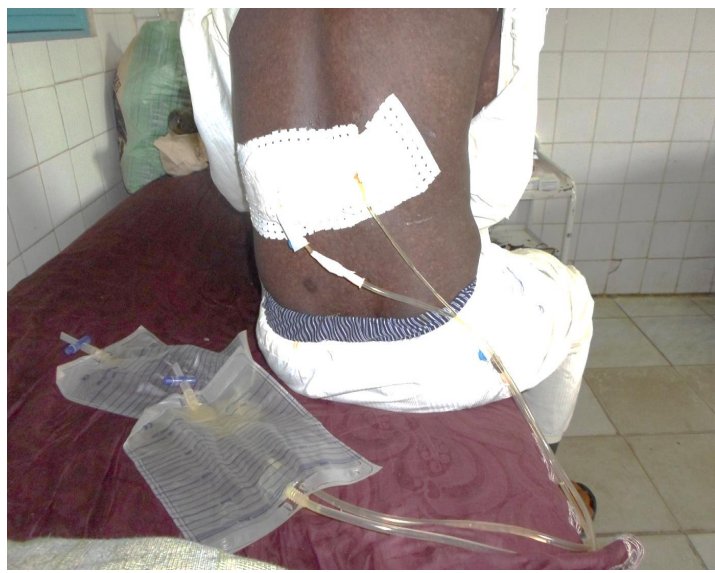

Figure 4. Ultrasound-guided bilateral nephrostomy (Urology Department of the NGRH, Chad).

Table 2. Distribution of patients according to surgical technique.

\begin{tabular}{ccc}
\hline Surgical Technique & $\mathbf{n}$ & $\%$ \\
\hline Pyelolithotomy & 11 & 39.3 \\
Nephrolithotomy & 7 & 25.0 \\
Ureterolithotomy & 3 & 10.7 \\
Ureterolithotomy + UVR & 2 & 7.1 \\
Nephrectomy & 5 & 17.9 \\
Total & 28 & 100.0
\end{tabular}




\section{Discussion}

\subsection{Epidemiological Data}

\subsubsection{Prevalence}

In our study, 1946 patients were consulted of which 101 consulted for renal colic, giving a prevalence of $5.19 \%$. This prevalence is comparable to that of the literature [1]. Our prevalence is statistically higher than that of LUJAN [6] in Spain, who reported $1.19 \%$. These differences could be explained by the hot climate, dehydration, mode of recruitment of patients, and especially the fact that the urology department of the NGRH is the only competent department in Chad capable of managing patients with renal colic.

\subsubsection{Socio-Demographic Data}

\section{1) Age}

According to the literature, renal colic mostly affects men of 20 to 60 years age group [1]. Our mean age of 38.69 years corroborates with these data. MUSTAPHA [7] in Turkey mentioned a young adult of middle age, and reported 31.1 years. This preferential involvement of young adults could be explained by the fact that urinary lithiasis mostly forms during the period of high sexual activity, which diverts all attention towards uro-genital infections. The prevalence of bilharzia infestation is high in infancy, and urological sequelae such as ureteral stricture and bladder tumors only occur many years later [8].

2) Sex

In our study, we found a male predominance with a sex ratio (male/female) of 1:3. From the existing literature, most authors unanimously agree on a male predominance [6] [9]. This male predominance could be explained by the anatomy of the male uro-genital system, which is prone to urinary stasis, and the role of oestrogens in women, which causes solubilisation of calcium by eliminating citrate [10].

\section{3) Month of occurrence}

We received most patients in the months of March, April and May as follows 9, 21 and 36 patients respectively. These are the hottest months in Chad. From the literature, dehydration predisposes to formation of stones and calculi. It is therefore possible that renal colic frequently occurs during hot and dry months [11]. EVANS et al. [12] have reported a high prevalence of renal colic in American soldiers deployed in Kuwait, a very hot country.

In our study, 64 patients (63.4\%) admitted to regularly consume milk and other dairy products. According to certain authors, milk is a risk factor for lithogenesis [11] [13].

\subsection{Clinical Aspects}

\subsubsection{Presentation of Complain}

Majority of our patients presented unilateral lumbar pain (92.1\%). The bilateral character of pain in our study could be explained by the high incidence/frequency of sequelae from bilharziasis and uro-genital tuberculosis. 


\subsubsection{Urological Antecedents}

Chronic urinary tract infection and urinary bilharziasis were the most common urological antecedents found at $18.8 \%$ and $6.9 \%$, respectively. KAMBOU et al. [3], in Burkina Faso reported $7.27 \%$ for chronic urinary tract infection and $54.54 \%$ for bilharziasis. Uro-genital infection is a risk factor for the formation of stones which is a major cause of renal colic. The high prevalence of urogenital bilharziasis in our countries could explain the majority of renal colics occuring without lithiasis.

\subsection{Paraclinical Aspects}

Uroculture revealed a urinary tract infection in $43.6 \%$ of cases in our study, Kabore et al. [14] reported $45.3 \%$. The variation in proportion of urinary tract infections in the different studies could be related to socio-economic conditions and access to health care. It is worth noting that Escherichia coli was the commonest germ in our study at $27.7 \%$. Ultrasound/KUB x-ray and intravenous urography made the diagnosis of lithiasis, stricture and ureteropelvic junction obstruction (SUJO) in 100\% since all the stones were radiopaque, and ultrasound showed ueretropyelocalyceal dilatation from strictures and SUJO. This sensitivity is comparable to PALMA [15], who reported $95 \%$.

The coupling of ultrasound/KUB X-ray for the diagnosis of lithiasis or pyeloectasia improves the individual performance of these tests and both furnish each other with complementary information [1]. In our study, urinary lithiasis was the most common etiology, found in 74 patients (that is $73.3 \%$ ), followed by ureteral stricture in 8 patients (that is 7.9\%). According to the existing literature, $90 \%$ of renal colic are caused by urinary lithiasis [16]. Our data corroborate with the existing literature.

\subsection{Therapeutic Aspects}

\subsubsection{Medical Treatment}

In our series, 73 patients $(72.3 \%)$ were treated medically. The remaining 28 patients $(27.7 \%)$ underwent surgery. It should be noted that most patients had micro-lithiasis of less than $6 \mathrm{~mm}$ in diameter. In our study, the mostly prescribed medical treatment consisted of a combination: NSAID + analgesic in 59 patients (that is 58.5\%). This corresponds to the recommendations of the 1999 consensus conference of the SFMU (Societe Francaise de Medecine d'Urgence), which recommends the prescription of NSAIDs except contra-indications associated with a first or second class analgesic depending on the intensity of the pain [5].

\subsubsection{Surgical Treatment}

Open surgery was performed in 28 patients (that are 27.7\%) using different techniques. Pyelolithotomy was performed in 11 patients (that are 39.3\%). This could explain the fact that open surgery still plays a major role as a means of stone extraction in developing countries. In most of these developping countries, sophisticated stone extraction techniques (ESWL, ureteroscopy, percutaneous 
nephrolithotomy [PCNL]) or other therapeutic approaches depending on the cause of renal colic are lacking.

\subsection{Evolution}

In our study, 57 patients (that are 56.4\%) healed without sequelae. TISELIUS [17] reported a higher success rate of $70 \%$ to $80 \%$. These differences could result from differences in technical platform as he used minimally-invasive surgery.

Thirty-three patients (that is $32.7 \%$ ) were lost to follow-up. This could account for the difficulty in follow-up and management of patients in our setting. Three patients left the hospital against medical advice, and two died.

\subsection{Limitations of Our Study}

The retrospective nature of our work was a limitation of this study, due to the insufficient follow-up of medical files. Some files could not be found, and among those found others were damaged. The information provided in the files was insufficient (summary clinical examination, history and long-term follow-up). The long-term evolution could not be assessed in any of our patients due to either insufficient records or the loss of sight of patients who did not return for follow-up consultation.

\section{Conclusion}

Renal colic is a common presented complain urology. Urinary lithiasis is a major etiology; but special attention should be placed on urinary schistosomiasis whose sequelae are a second leading cause. Treatment must be initiated early to prevent complications; however, modern techniques such as endoscopy and double Catheter stenting should be used to improve on the management of complicated forms of renal colic.

\section{Conflicts of Interest}

The authors declare no conflicts of interest regarding the publication of this paper.

\section{References}

[1] El Khebir, M., Fougeras, O., Gall, C., Santin, A., Perrier, C., Sureau, C., et al. (2008) Update of the 8th Consensus Conference of the French-Speaking Medical Emergency Society of 1999. Management of Renal Colic Adults in Reception and Emergency Services. Progrès en Urologie, 19, 462-473. https://doi.org/10.1016/j.purol.2009.03.005

[2] Tour, C.T. and Dieng, M. (2002) Emergencies in a Tropical Environment: Inventory, the Example of Surgical Emergencies in Senegal. Medecine Tropical, 62, 237-241.

[3] Kambou, T., Traoré, A.C., Zongo, B., Bonkoungou, B., Ouattara, T. and Sanou, A. (2005) Lithiasis of the Upper Urinary Tract at CHU of BOBODIOULASSO (Burkina Faso): Epidemiological, Clinical and Therapeutic Aspects: About 110 Cases. African Journal of Urology, 11, 55-60.

[4] Gasma, D. and Abdou, C.C. (1996) Nephretic Colic. Annales d Urologie, 30, 276-277. 
[5] Touze, M.D., Bertini, N., Ducasse, J.L., Ellrodt, A., Gattegno, B., Guille, F., et al. (1999) Management of Nephritic Colic in the S.A.U. SFMU Consensus Conference. 4-M-10.

[6] Luján, M., Sánchez, M.T., Turo, J., Pascual, C., Chiva, V., Martín, C., et al. (2011) Climate and Epidemiological Characteristics of Renal Colic Attendances in an Urban Setting in Spain. Actas Urológicas Españolas, 35, 481-486. https://doi.org/10.1016/j.acuro.2011.03.007

[7] Mustafa, S., Ozgur, K., Ibrahim, T., Halis, O., Mustafa, K., Aytaç, B., et al. (2008) Analysis of Clinical and Demographic Characteristics of Patients Presenting with Renal Colic in the Emergency Department. BMC Research Notes, 1, Article No. 79. https://doi.org/10.1186/1756-0500-1-79

[8] Giannakopoucos, X., Evengelou, A., Soumanis, P., et al. (1996) Urinary Tract Infection in Lithiasis in the Department of Epius (North West Ridge). Annales d Urologie, 30, 118-123.

[9] Marcia, L., Justin, W., Robert, J., Sedran, M.D., Shelley, L. and McLeod, M.S. (2010) The Utility of Renal Ultrasonography in the Diagnosis of Renal Colic in Emergency Department Patients. Canadian Journal of Emergency Medicine, 12, 201-206. https://doi.org/10.1017/S1481803500012240

[10] Green, J.H. (1984) Fluid and Electrolyte Balance and the Kidney. In: Green, J.H., Ed., Manual of Clinical Physiology, 2nd Edition, Edition Masson, Paris, 109-124.

[11] Mahamat, A.M., Ngaringuem, O., Mahamat-Nou, A., Jallot, M., Niang, L., Hamat, I., et al. (2017) Low Urinary Tract Lithiasis: Diagnostic and Therapeutic Aspects (HME) in N'djamena (Chad). African Journal of Urology, 23, 295-299. https://doi.org/10.1016/j.afju.2016.11.003

[12] Evans, K. and Costabile, R.A. (2005) Time to Development of Symptomatic Urinary Calculi in a High Risk Environment. Journal of Urology, 173, 858-861. https://doi.org/10.1097/01.ju.0000152578.07262.1c

[13] Sakly, R. and Achour, A. (1991) Dietary Approach to Renal Lithiasis. Annales d'Urologie, 25, 237-241.

[14] Kaboré, F.A., Kambou, T., Zango, B., Ouattara, A., Simporéa, M. and Lougué, C. (2013) Epidemiology of a Cohort of 450 Urinary Lithiasis at the CHU Yalgado Ouédraogo in Ouagadougou (Burkina Faso). Progress in Urology, 23, 971-976. https://doi.org/10.1016/j.purol.2013.04.014

[15] Palma, L.D., Stacul, F., Bazzochi, L., et al. (1993) Ultrasonography and Plain Film versus Intravenous Urography in Ureteric Colic. Clinical Radiology, 47, 333-336. https://doi.org/10.1016/S0009-9260(05)81449-7

[16] Houlgatte, A. and Deligne, E. (2007) Cholic Nephretic. EM Elsevier Masson SAS, Paris.

[17] Tiselius, H.G., Alken, P., Buck, C., Gallucci, M., Knoll, T., Sarica, K., et al. (2008) Guidelines on Urolithiasis: Diagnosis Imaging. European Urology EAU Guidelines, 9-19. 